Horizons philosophiques

\title{
L'identité européenne : un débat qui met en lumière les difficultés conceptuelles de l'identité
}

\section{Aude-Emmanuelle Fleurant}

Volume 11, numéro 2, printemps 2001

L'Europe, le laboratoire d'idées du XXI ${ }^{\mathrm{e}}$ siècle?

URI : https://id.erudit.org/iderudit/801182ar

DOI : https://doi.org/10.7202/801182ar

Aller au sommaire du numéro

Éditeur(s)

Collège Édouard-Montpetit

ISSN

1181-9227 (imprimé)

1920-2954 (numérique)

Découvrir la revue

Citer cet article

Fleurant, A.-E. (2001). L’identité européenne : un débat qui met en lumière les difficultés conceptuelles de l'identité. Horizons philosophiques, 11(2), 58-89.

https://doi.org/10.7202/801182ar d'utilisation que vous pouvez consulter en ligne. 


\section{L'IDENTITÉ EUROPÉENNE : UN DÉBAT QUI MET EN LUMIÈRE LES DIFFICULTÉS CONCEPTUELLES DE L'IDENTITÉ}

\section{L'Union européenne et la redéfinition de l'identité collective : le débat}

L'Union européenne (UE) a suscité, depuis son émergence, de multiples bouleversements dans l'organisation politique et économique des États membres. En effet, les diverses mesures mises en place, telles la libre circulation de la main d'oeuvre, l'abolition des douanes, les programmes de convergence et d'harmonisation économiques pour les régions ou encore récemment, l'implantation du statut de "citoyen européen", tout en modifiant considérablement la configuration économique et politique des États concernés, semblent accélérer le mouvement vers une fusion plus importante au plan politique. Cette situation sans précédent et sans corollaire sur la scène internationale engendre des débats et des questionnements importants quant à la légitimité des décisions et des actions entreprises ou encore quant à la présence et à la qualité de l'exercice démocratique au sein de l'Union.

II apparaît évident que le processus de construction européenne, outillé d'un projet de plus en plus concret d'intégration politique, comprend un nombre important d'enjeux. Que ce soit en termes de définition d'une sécurité et d'une politique extérieure proprement européennes, que ce soit en termes de contrôle de l'immigration ou encore en ce qui concerne l'élargissement même de l'Union, on s'interroge sur la façon de concevoir et d'opérationaliser une Europe à la fois ouverte, démocratique et vraiment représentative des intérêts de ses diverses composantes. Bref, il semble qu'à l'heure actuelle, le défi consiste à créer un espace politique commun et démocratique permettant aux Européens de se prononcer sur les enjeux qui les concernent.

Le débat entourant l'apparition d'une identité européenne s'inscrit dans la foulée de ces réflexions, puisqu'il s'agit de définir ce que signifie être européen et d'indiquer qui peut s'en réclamer. Toutefois, cette discussion nous paraît également vouloir dépasser le strict périmètre de la problématique de l'unification politique du conti- 
nent, situation éminemment nouvelle qui, par là, peut introduire la nécessité d'un exercice de positionnement des collectivités par rapport à l'entité en construction. Dans ce contexte, s'avère préoccupante la question jamais résolue des conditions d'émergence, des fondements et du rôle de l'identité nationale dans les sociétés occidentales modernes ainsi que les origines du phénomène de crise qui lui est actuellement associée, notamment dans les "vieux» Étatsnations'.

Identities are being redefined as individuals, cities, regions and states come to terms with the dynamics of the "new Europe". Uncertain identities at the national and local level feed into debates about the desirability and feasability of a European identity which may be essential if the European Union is to enhance its legitimacy and become a genuine political realm (Laffan 1996, p. 83).

Pour plusieurs, l'Europe offrirait donc un cadre inédit pour la construction d'une identité qui pourrait se détacher des termes qui définissent habituellement les identités nationales. Cependant, le processus d'intégration européen est perçu selon divers angles et des lectures différentes de ce phénomène et de son influence sur les identités locales, régionales et nationales amènent divers auteurs à formuler et à présenter des conceptions hétérogènes de ce en quoi pourrait consister la nouvelle identité européenne, laissant parfois le lecteur perplexe.

Pour certains, l'intégration politique doit s'accompagner de la naissance d'un sentiment d'appartenance (construit ou spontané) dont la teneur se rapprocherait de celui suscité par la nation. Pour d'autres, la nouvelle Europe permettrait un remodelage de l'identité à partir de fondements inédits, évitant ainsi les limites d'une exclusion estimée intrinsèque aux identités nationales ou ethniques. II ressort de ces propositions divergentes sur la question que la discussion actuelle sur l'identité européenne, s'intéressant tout autant à la question de la formation d'une identité politique ou culturelle qu'à la résolution des crises identitaires contemporaines (Lemke 1998, p. [2]), s'avère extrêmement riche au plan théorique, mais demeure confuse et peut paraître éclatée.

C'est pourquoi nous avons décidé de brosser un portrait général de ce débat, dont le principal objectif est de procéder à une revue des positions marquantes identifiées dans la discussion en cours sur

1. Mentionnons entre autres, les crises d'identité anglaise et française qui sont toutes deux amplement discutées actuellement dans la littérature scientifique, notamment en regard des velléités autonomistes de l'Écosse ou de la Corse. 
l'identité européenne afin d'offrir à la lectrice et au lecteur une grille de lecture fidèle à la complexité de la situation. La littérature sur le sujet étant abondante, nous avons toutefois choisi de circonscrire le débat dans le temps en nous appuyant essentiellement sur ce qui a été écrit depuis la fin de la guerre froide, à l'exception notable du texte de Raymond Aron, datant de 1974, mais qui s'est révélé fort pertinent à notre propos.

Nous estimons que la discussion autour de l'émergence ou de la construction d'une identité européenne et de sa signification a pris de l'ampleur depuis la chute du mur de Berlin, événement qui a forcé, en quelque sorte, les Européens de l'ouest à redéfinir cet "autre» contre lequel l'Europe d'après-guerre s'était modelée. Par ailleurs, le débat actuel semble surtout s'articuler autour de problématiques particulières. À ce titre figurent d'abord des phénomènes qui sont perçus comme très inquiétants : la montée de l'extrême droite dans la plupart des pays membres de I'Union (France, Autriche, Allemagne, etc.) ainsi que la multiplication des crises dites "ethniques " dans les États voisins d'Europe de l'Est. S'ajoutent ensuite les difficultés de la mise en place du Traité de Maastricht. Bref, en général, la réflexion sur la construction identitaire s'effectue à partir d'un contexte qui serait jonché d'obstacles. C'est pourquoi nous estimons que certaines des approches présentées comportent des prises de positions idéologiques (explicites ou implicites) face à ces phénomènes et face au type de nationalisme qui leur est généralement associé, prises de position que nous tenterons de mettre en lumière tout au long de la démarche.

II ne s'agit pas de faire ici un état de la question exhaustif. Le débat est récent et en constante évolution. Nous souhaitons plutôt proposer à l'intéressé-e une grille de compréhension de la discussion qui mette en lumière les divers postulats implicites à chacune des approches présentées et qui relève les critiques et interrogations que posent les objectifs poursuivis par chacune des "écoles de pensée" en la matière. La pertinence de cet exercice réside dans l'établissement de points de repères importants mais aussi de limites pour un débat riche où foisonnent concepts et idées.

Nous tenons à spécifier que cet article ne prétend pas non plus rendre justice à la variété des idées proposées en matière d'identité européenne. Cependant il porte sur les propositions qui paraissent rallier le plus d'adeptes et être le plus discutées en ce moment. C'est pourquoi en conclusion, nous effectuerons un 
commentaire général sur l'exercice où nous présenterons les pistes de recherche intéressantes qui se seront révélées en filigrane tout au long de l'élaboration de la synthèse-critique.

\section{Les fondements du débat et la perspective d'une intégration poli- tique}

Tout d'abord, il nous semble important de mentionner qu'il s'agit d'un débat à caractère très académique et qu'il semble surtout circonscrit aux milieux intellectuels. L'enthousiasme généré par la problématique et l'aspect parfois très prescriptif du langage utilisé dans les publications ne devraient pas occulter le fait qu'à quelques exceptions près, on ne souhaite pas implanter ou faire surgir concrètement une identité européenne mais surtout en discuter ou en faire progresser l'idée. En bref, nous ne croyons pas qu'il soit proposé de recette de l'identité. C'est simplement que l'Europe, cet objet politique non-identifié (pour reprendre la terminologie de Jacques Delors) constitue un terrain fertile de questionnements et de confrontation à propos de tout ce qui touche, entre autres sujets, la crise de l'État-nation, l'érosion de la souveraineté des États, le nationalisme et l'identité. À cet égard, certains éléments semblent faire consensus dans la littérature consultée jusqu'à présent.

Dans un premier temps, nous avons relevé que les protagonistes du débat le situent dans la perspective où le projet européen en serait à une "étape critique" de son développement. Cette notion semble en fait vouloir indiquer l'existence de deux phénomènes distincts : l'inéluctabilité de l'intégration politique et le manque de légitimité de l'Europe comme entité politique pour parvenir à cette unification.

D'abord, on tend à présenter l'intégration politique comme quelque chose qui serait en retard sur le processus d'intégration économique.

The third problem is the potential for the "democratic deficit", from which the Community already suffers, becoming acute if institutional and political reforms continue to lag behind the creation of a single market and moves towards economic and monetary union (Bull 1993, p. 40).

Cette vision très téléologique de la construction de l'Europe pose, selon nous, des liens un peu mécaniques entre intégration économique et intégration politique, liens qui sont peu remis en question dans la littérature. Dans cette perspective, on semble accepter d'emblée et sans la questionner l'idée que l'intégration politique soit 
nécessaire, et on stipule que sa concrétisation doit se faire via une forme d'identification individuelle et/ou collective à un hypothétique espace politique représenté par une communauté qui reste à définir, mais qui prend son origine dans des stratégies économiques.

On peut également associer à cette schématisation de l'évolution de l'Europe qui en serait à l'étape de crise, l'idée assez répandue que le projet fonctionnaliste-néofonctionnaliste (souvent présenté sous le nom de projet Monnet) situé à l'origine de l'Europe, aurait atteint ses limites intrinsèques et qu'il est désormais nécessaire de les dépasser et ce faisant, de redéfinir le projet européen (Featherstone 1994, p. 165). D'une part, cela nous paraît être plus une affirmation rapide qu'une constatation formelle ou même empirique du lien existant entre les divers obstacles actuels au bon fonctionnement de l'Europe et la façon dont cet ensemble d'institutions aurait été initialement pensé et implanté. D'autre part, cette affirmation tend à donner l'impression que les objectifs, les structures et le fonctionnement de l'Europe, malgré la fin de la guerre froide, malgré les multiples étapes telles Maastricht ou Schengen, malgré les "élargissements et les approfondissements", seraient demeurés immuables, ne se seraient pas adaptées à de nouvelles aspirations des sociétés ou à un nouvel environnement géopolitique, économique et social.

De plus, cette manière de présenter les choses tend à gommer le fait que la construction de l'Europe ne s'est pas faite de façon linéaire et stable, comme le rappelle Abelès (1996), mais qu'en fait, elle a été marquée par d'importantes périodes de stagnation et de crises que l'auteur qualifie de "temps morts" et où, à l'instar du débat actuel, on annonçait soit l'échec du projet soit la nécessité de réformes majeures pour assurer sa pérennité.

Par ailleurs, il semble important de souligner qu'il y a dans les prémisses présentées plus avant une position implicite quant à une autre discussion portant justement sur l'opportunité d'un "approfondissement" politique de l'Union, question qui ne fait pas véritablement consensus actuellement. On peut se demander quelle serait la pertinence, voire l'utilité, d'un questionnement sur l'identité européenne sans l'acception de l'idée d'une intégration politique dont on souhaite activement l'imminence.

En second lieu, la notion d'étape critique est soutenue par l'idée qu'il y aurait en Europe une crise étroitement associée au manque de légitimité des institutions européennes. La principale cause de cette 
crise serait un déficit démocratique important et l'un de ses principaux symptômes serait le manque d'intérêt des populations face aux affaires européennes, notamment face aux élections parlementaires européennes.

Les notions de déficit démocratique et de crise politique de l'Europe sont souvent présentées en appui à l'idée de la nécessité d'une forme d'identification à l'Europe, identification qui constituerait une solution partielle mais essentielle au problème de légitimité. Toutefois, le lecteur est en droit de se demander si le déficit démocratique de l'Europe constitue véritablement un élément d'une crise politique fondamentale ainsi qu'une pierre d'achoppement significative au bon fonctionnement de l'Europe ou si cette argumentation n'est pas plutôt, de manière un peu circulaire, une façon de poser la nécessité de l'identité européenne face à une intégration politique qui apparaît, dans la plupart des textes, comme la prochaine étape inéluctable du développement européen.

Pour continuer dans cette veine, Hensen et Williams (1999) proposent une lecture différente du phénomène en relevant tout d'abord que le débat autour de la légitimité de l'Europe apparaît, depuis quelques années, nettement plus axé sur les dimensions de la démocratie et de la citoyenneté plutôt que sur les liens intergouvernementaux qui ont longtemps été les fondements de la légitimité de la Communauté. Toutefois, pour ces auteurs, ce glissement dans le débat peut s'expliquer par le fait que le caractère de plus en plus supranational et "ingérent" des institutions européennes aurait créé chez les populations une opposition plus globale à l'Europe, contribuant à l'identification de lacunes au plan de la démocratie comme étant la cause de la crise et par le fait même, la façon de remédier à la situation.

Scholars and politicians who diagnose the current crisis in these terms direct attention to the relationship between governments and the EU on the one side and the electorates on the other, not as has normally been the case in European integration studies, to intergovernmental studies. This construction of the crisis turns the discussion towards questions of legitimacy and democracy, and more specifically, asks how improving the latter can alleviate the scepticism towards the EU. (Hensen et Williams 1999, p. 234-235).

Dans cette perspective, la notion de crise orienterait le débat entourant l'intégration politique en mettant en valeur sa nécessaire 
concrétisation comme moyen privilégié de résoudre la crise. L'idée d'une crise démocratique de l'Union ne serait donc pas innocente et pourrait être révélatrice d'une position implicite de certains auteurs dans le débat opposant ceux qui sont "pour" et ceux qui sont "contre" l'Europe et non pas, pour "plus ou moins" d'Europe, comme c'était généralement le cas auparavant.

Voici donc, succinctement, les principaux éléments qui paraissent faire consensus dans la littérature récente sur la question de l'identité européenne. Ces diverses prémisses éclairent le débat sur l'identité européenne en situant ses frontières ainsi qu'en permettant de dégager le contenu des idées proposées de façon critique.

\section{Les quatre grandes approches}

\section{L'identité postnationale : raison et citoyenneté}

Pour plusieurs auteurs, l'originalité et la nouveauté de l'espace politique qui est en train de se créer autour des institutions européennes fournit l'occasion de proposer une nouvelle forme identitaire qui se fonderait sur des éléments qu'ils estiment totalement différents de ceux constituant l'identité nationale, comme la culture, la langue ou le sentiment d'appartenance à une communauté. On soutient que ce "type" d'identité n'est ni opérationnalisable, ni souhaitable dans un contexte européen avant tout marqué par son caractère pluraliste et diversifié, tant au plan des valeurs qu'au plan des coutumes ou des pratiques socio-politiques et économiques. On part de la prémisse largement acceptée dans la communauté scientifique que l'identité, comme la nation, est un construit et qu'il est donc possible de la concevoir autrement qu'elle n'a été définie jusqu'à présent et en particulier dans le cadre national.

European identity did not exist prior to its definition and codification. It is a doubtful construct anyway given the apparent irresolvable conflict of national cultures and oppositional collective identities. Unifying myths of integration should be viewed with scepticism unless they unambivalently accommodate diversity (Delanty 1995, p. 3).

La sauvegarde du caractère pluriel de l'Europe apparaît donc être la préoccupation centrale et le point de départ de toute la réflexion des tenants de l'approche postnationale qui, par ailleurs, présentent les problèmes liés à l'immigration comme la preuve de l'achoppement d'une définition identitaire conforme à des critères nationaux. Puisque 
les identités nationales sont actuellement incapables d'accommoder convenablement le multiculturalisme croissant (à cause de l'immigration) des États occidentaux, quelle serait la performance d'une seule identité supranationale restrictive conçue en fonction de paramètres semblables? (Habermas 1995, p. 271-277). Par ailleurs, la nouvelle européanité ne devrait pas supplanter, ni diluer, les autres allégeances, au contraire, elle devrait être en mesure de mieux les préserver que ne le font les identités nationales (Graham 1998).

Pour poursuivre cet objectif, les auteurs s'interrogent sur la façon de constituer un espace politique différent, fondé sur des valeurs qui dépasseraient les limites de l'identité nationale et qui permettraient à la fois l'inclusion de tous dans la communauté ainsi que la préservation des diverses cultures. La réponse postnationale, prenant largement ses appuis et son inspiration dans les travaux de Habermas portant sur le citoyenneté et l'intersubjectivité, propose donc une identité qui se formule en termes d'allégeance rationnelle et individuelle à des principes universels formalisés au sein d'une constitution. Idéalement, cette constitution devrait être le principal référent identitaire des individus, ce qui vaut également à l'approche postnationale le nom de "patriotisme constitutionnel». On rejette ainsi toute forme d'identité qui serait fondée sur des critères considérés comme douteux ou dangereux tels l'appartenance ethnique ou culturelle.

L'identité nationale ne désigne pas d'abord et avant tout une échelle supranationale de citoyenneté. Elle désigne avant tout un motif non nationaliste de participation à une communauté politique (...) II s'agit d'un attachement politique qui se détermine non pas sur des critères de co-appartenance ethnique, linguistique ou culturelle, mais sur des critères éthiques de reconnaissance réciproque des sujets de droits, individus ou États, ainsi que sur les critères politiques d'une reconnaissance commune de principes fondamentaux tels que ceux de la démocratie et de l'État de droit (Ferry 1995, p. 92).

Est donc suggérée l'émergence d'une communauté de volonté située exclusivement dans la sphère politique, par opposition à une certaine conception de l'identité collective qui postule l'entretien de liens ou d'une certaine congruence avec une communauté préexistente, que celle-ci soit un construit ou qu'elle représente une réalité objective. La communauté politique européenne est ainsi imaginée comme une agrégation ouverte et volontaire d'humains égaux et rationnels se reconnaissant eux et les autres comme sujets universels (Booth 1999). II n'y a pas de nation européenne, il n'y a que des Européens. D'ailleurs, le statut de citoyen européen constitue une 
pierre d'assise de l'élaboration de l'identité européenne. Cette citoyenneté, définie dans ce cadre comme étant la relation entre l'individu et la communauté politique (Wiener et Della Sala 1997, p. 601), serait ouverte et accessible à tous ceux et celles qui désireraient s'en prévaloir, puisque la communauté politique, épurée de tout sentiment, serait par essence complètement ouverte.

II est intéressant de remarquer que les postnationalistes s'inscrivent clairement en porte à faux avec deux grandes théories explicatives de l'identité et de la nation, soit celle de Gellner d'une part, qui avançait l'idée d'une certaine adéquation entre communauté (nation) et État et celle de Smith d'autre part, pour qui l'appartenance ne peut se définir en termes exclusivement rationnels, ce sur quoi nous reviendrons plus loin.

Conception hautement idéaliste de l'admission même des auteurs qui la défendent, l'approche postnationale représente une charge virulente contre les nationalismes destructeurs, notamment ceux apparus en Europe de l'Est depuis la chute des régimes communistes. On reproche globalement au nationalisme ses excès et son potentiel de violence à l'égard de la différence, mais, de façon plus significative, on situe les germes du nationalisme comme mouvement politique répressif dans le phénomène émotif d'identification nationale. Dans cette optique, un des objectifs visé par l'école postnationale se révèle comme étant l'élimination de la dimension affective de l'identification politique, dimension qui est présentée comme une des causes principales des divers conflits violents qui ont marqué l'histoire contemporaine du continent européen et qu'on cherche à tout prix à éradiquer pour de bon. La rationalité de la pratique politique est donc présentée comme la seule façon de préserver à la fois la paix et la diversité culturelle sur le territoire européen.

Parlant du nouveau "citoyen européen» John Keane s'avance encore plus et affirme que (...they) maintain that in the contemporary world identity is more a matter of politics and choice than fate. They have an allergic reaction to nationalism and deep empathy for people suffering discrimination or enforced exile from their cherished nations or territories (Keane 1995, p. 205).

Ce qu'il faut retenir de l'approche postnationale se résume fondamentalement en deux choses. Dans un premier temps, son projet identitaire, qui ne peut se concrétiser qu'à travers l'avènement d'une citoyenneté européenne, repose sur l'adhésion volontaire et rationnelle à un ensemble de grands principes démocratiques 
fondés essentiellement sur le droit. Cette approche suggère donc une sorte de retour à la société civile ou au contractualisme envisagé pendant la période des Lumières, mais dans un espace public totalement différent de celui de l'État-nation. Dans un deuxième temps, cette définition de l'identité suppose l'instauration d'un système de valeurs qui rejetterait dans son ensemble les dimensions émotives et nationalistes de la politique.

\section{Un projet utopique qui se pose contre le nationalisme}

Une interrogation majeure qui surgit à la lecture concerne les éléments de définition de l'identité européenne qui y sont avancés. L'approche postnationale offre d'abord et avant tout une conception de la communauté politique basée sur l'addition de ses membres, eux-mêmes définis par leur statut de citoyen, c'est-à-dire par leurs droits et leurs devoirs légalement établis. D'une part, la priorité accordée à la dimension individuelle nous paraît une façon hautement difficile et hasardeuse de comprendre et d'articuler une notion telle que celle de communauté politique. D'autre part, on suppose également a priori que les membres volontaires et implicitement actifs de cette communauté seront rationnels et que s'ils le sont, ils adhéreront nécessairement aux principes universels contenus dans la constitution. II s'agit donc, à tout le moins, d'une façon très utopique d'envisager la future communauté européenne.

Bien que leur teneur soit tout à fait pertinente, nous ne mentionnerons pas les critiques des relativistes quant à l'universalité supposée du droit et de la rationalité occidentale, ainsi que leur valeur intrinsèque en tant que garde-fou de la démocratie et de la tolérance, dimensions qui sont systématiquement mises de l'avant par les postnationaux. Même si cette discussion pourrait s'avérer fort profitable, ce serait sortir du débat sur l'identité européenne stricto sensu. Par contre, nous ne pouvons faire l'économie de toutes les critiques portant sur la préséance de la raison et du droit comme fondement identitaire et une question centrale se pose quant aux possibilités concrètes de mise en oeuvre de l'identité postnationale qui en découlerait. En effet, comment stimuler une volonté de participation politique de la part des individus membres en faisant exclusivement appel à la raison et au droit? La solution préconisée par les tenants de cette approche est, invariablement, celle d'une nouvelle citoyenneté qui ramène à l'avant-plan de la pratique démocratique l'essence des grands principes de la relation de droits et de devoirs État-citoyen élaborés lors de la période des Lumières (Habermas 1995; Leca 1991). 
Or, la définition donnée à la citoyenneté européenne par les postnationalistes est remarquablement similaire à celle qu'ils attribuent également à l'identité, c'est-à-dire que la citoyenneté, tout comme lidentité, est fondée sur l'adhésion volontaire et rationnelle à des principes de droits démocratiques et universels qui seraient inscrits dans une constitution (Delanty 1995, p. 159-160).

Citizenship is an answer to the questions "Who am I?" and "What should I do?" when posed in the public sphere. Membership in a political community confirms special duties, behind which stands a patriotic identity (Habermas 1995, p. 276).

Les auteurs semblent donc fondre ensemble les notions d'identité et de citoyenneté. Cependant, il nous semble particulièrement réducteur de définir l'identité - même si on la situe exclusivement dans le champ politique - par la citoyenneté. Pour illustrer simplement cette difficulté, prenons l'exemple d'un militant écologiste. Son identification et sa participation au mouvement peuvent s'être établies sur la base de critères rationnels et universels (réduction objective de la quantité de ressources énergétiques, sauvegarde des grands systèmes écologiques pour le maintien de l'équilibre planétaire, etc.) II s'agit peut-être même de son premier référent identitaire. II n'a pourtant pas de statut de "citoyen écologiste". Le même exemple peut être appliqué aux féministes, aux ouvriers ou à tout autre membre d'un mouvement social qui milite sur la scène politique. Cette personne peut même, au nom de ses principes rationnels, se prévaloir d'un de ses droits de citoyen (le droit de vote) dans l'optique très nette d'infléchir le politique en fonction de ses propres convictions. Par ailleurs, sa citoyenneté nationale ne l'empêche pas de se concevoir d'abord comme écologiste, féministe, pacifiste etc., et cette identité dominante peut même l'amener à défier l'État dont il est le citoyen (désobéissance civile), le tout, encore une fois, sur la base d'un calcul rationnel.

Une autre remarque qui vient à l'esprit à la lecture des textes sur l'identité postnationale est la confusion qui apparaît entre identité nationale et nationalisme. Très peu d'efforts de définition et de distinction entre ces deux concepts sont faits par les auteurs qui rejettent en bloc toute forme de sentiment d'appartenance culturelle, qui est considérée a priori comme irrationnelle et exclusive. Cette prise de position sans équivoque révèle cependant que les postnationalistes ont, implicitement, une perception de l'identité nationale et du nationalisme qui est non seulement très négative, mais aussi à notre avis, réductrice. 
En opposant systématiquement une identité européenne fondée sur la rationalité du sujet ainsi que sur un espace de participation démocratique avec l'identité nationale fondée sur des critères ethniques considérés comme discutables, on en vient à donner l'impression que les identités nationales sont essentiellement irrationnelles, fermées, antidémocratiques et discriminatoires et que ce sont ces dimensions moralement répréhensibles qui ont donné naissance aux nationalismes haineux et violents que l'Europe a connu. II s'agit, selon nous, d'une façon extrêmement sélective d'examiner le rôle du nationalisme dans l'histoire moderne et contemporaine à la fois de l'Europe et d'autres parties du monde. II ne faudrait pas oublier que pour nombre de nations, dont la France et les États-Unis, le passage de sujet du royaume à citoyen porteur de droits a d'abord été le fait de démocraties nationales.

Laisser la nation à l'extrême droite, c'est lui faire un superbe cadeau. Et c'est aussi faire une croix sur presque un siècle d'histoire politique. En effet, de la Révolution française aux années 1880 , le concept de nation a été de tous les combats des libertés contre l'Ancien Régime.(...) Le phénomène le plus dangereux consiste à établir des signes d'égalité entre nation, sentiment national, idéologies nationalistes et nationalisme (Wolton 1993, p. 100-101).

Nous aimerions, en dernier lieu, éclairer ce que nous considérons comme une dimension réductrice de l'approche postnationale. Nous estimons qu'en ayant comme valeur centrale et comme pierre d'assise de toute l'analyse la sauvegarde et le maintien du pluralisme des sociétés européennes, les auteurs limitent en fait la problématique de l'identité européenne et de l'unification politique à cette unique dimension. Par ailleurs, n'est-il pas un peu ambigu de préconiser la sauvegarde du multiculturalisme par une absence totale - voire un rejet - de toute forme de participation culturelle au politique? N'est-il pas en fait, un peu paradoxal de définir l'Européanité en procédant d'abord et avant tout à l'énumération de ce qu'elle ne doit pas être?

\section{Un État supranational européen, une civilisation européenne.}

Les auteurs s'inscrivant dans cette approche se situent eux aussi dans la perspective de l'imminence de la création d'un nouvel espace politique qui pourrait remettre en cause, du moins partiellement, les définitions nationales de l'identité et de l'appartenance. Mais leurs préoccupations centrales diffèrent substantiellement de celles de leurs collègues postnationaux. Le principal défi à relever dans le cas des 
supranationaux semble être une reformulation des concepts de souveraineté et d'auto-détermination par rapport à d'autres paramètres que ceux de l'État-nation, ce qui correspondrait en fait plus à la réalité actuelle de l'Europe. En effet, la plupart des tenants de l'approche supranationale partent de l'observation que le système européen a considérablement compromis la souveraineté des États-membres.

Students of European integration and international politics struggle to characterize a state system in which constitutional independence has thus been ceded, sovereign equality modified, economic autonomy long since deeply compromised, security managed through an integrated alliance, internal borders opened and external borders managed through a common regime, monetary sovereignty shortly to yield to a common currency (Wallace 1999 p. 518).

Aborder la problématique de l'identité européenne sous cet angle place inévitablement au centre du questionnement la légitimité de ce nouveau système, ainsi que la représentativité des institutions qui en découlent et qui ont contribué à modifier substantiellement le paysage politique européen. C'est donc la question de l'approfondissement de l'Union qui prédomine pour les adeptes de l'approche supranationale, plus que celle de son élargissement (Garcia 1993, p. 3).

Dans l'optique adoptée par les auteurs de l'école supranationale, la naissance d'une conscience européenne est une composante essentielle d'un processus qui démocratiserait l'Europe et qui pourrait permettre ultimement aux populations européennes de donner leur aval aux actions entreprises. Le système européen est ici largement perçu comme étant particulièrement opaque, inaccessible et également comme étant gouverné par une élite technocratique détachée des besoins des populations. Ces dernières auraient par ailleurs clairement affiché leur mécontentement face à cette situation lors des référendums quant à l'adoption du traité de Maastricht, introduisant ainsi la crise actuelle de l'Europe (Reik 1993, pp. 148-149).

L'apparition d'une identité européenne constitue donc une partie de la solution à cette crise. Mais contrairement à ce qu'avançait l'approche postnationale, les supranationaux semblent estimer qu'on doit s'efforcer de mettre en valeur ce qui est commun au sein de la diversité européenne pour qu'une européanité puisse réellement se dessiner et se concrétiser.

It has been pointed out (...) that a certain level of common identity is required in order to legitimize the existence and further development of European integration. (Reik 1993, p. 131). 
Ainsi, l'approche supranationale tente de surmonter la barrière que représenterait la diversité culturelle à l'élaboration d'une identité européenne et surtout, à l'émergence d'un sentiment d'appartenance à une nouvelle collectivité. Toutefois, ceux qui soutiennent ce point de vue n'attribuent rien de naturel à une éventuelle identité européenne. Les auteurs tenants de l'approche supranationale estiment qu'une conscience européenne - pour reprendre une terminologie qu'ils privilégient - peut être construite, mais cela à partir de plusieurs éléments d'un passé commun largement partagé par les sociétés européennes.

On s'accorde généralement pour discerner un "fonds culturel européen" qui serait issu d'un mélange plus ou moins bien défini d'hellénisme, de loi romaine, de judéo-christianisme ainsi que d'héritage des Lumières et des romantiques (Wolton 1993, p. 129) ou des diverses crises politiques qui ont égrené l'histoire contemporaine de l'Europe (Gireault 1994, p. 194-195). Ce sont ces ingrédients qui, grosso modo, permettraient d'affirmer l'existence d'une certaine forme d'européanité (ou d'une civilisation européenne) sur laquelle l'identité collective des européens pourrait se fonder et croître.

Dans la perspective adoptée par les tenants de l'école supranationale, la dimension affective est réintégrée dans la définition de l'identité collective, sans toutefois la réduire à cet unique aspect, l'identité comprenant son lot d'histoire, de principes rationnels et de nécessités objectives. Néanmoins, le fait est que le sentiment d'appartenance est considéré comme un élément essentiel de l'identité européenne, elle-même nécessaire à la concrétisation de l'unification politique ainsi qu'à la résolution de la crise.

Political communities are based not just on rational calculations but on sentiment, solidarity and a degree of political cohesion. (...) Although Europe cannot create a demos in the traditional sense, some actors in the integration project have stressed the state-building and community-building aspects of their joint enterprise. The need to create a "people's Europe" and to strengthen the public's identification with the European project has been a recurring theme in official thinking on European integration (Laffan 1996, pp. 95-96).

Se démarquant de l'approche postnationale, les supranationaux n'opposent pas une éventuelle identité européenne à l'identité nationale, mais, de façon plus explicite, aux nationalismes d'extrême droite. Une véritable conscience communautaire permettrait de consolider les grands principes démocratiques et étatiques de droit 
qui gouvernent l'Europe et les acteurs pourraient ainsi formuler une réponse "typiquement européenne" aux problèmes associés aux mouvements nationalistes radicaux et aux mouvements ethnonationaux. II semble important de noter que cette réflexion prend ses racines dans l'impuissance de l'Europe à intervenir avec efficacité et discernement dans la crise yougoslave, un État au statut ambigu, qu'on situe, au demeurant, à l'extérieur de l'Europe, définissant du même coup et plus ou moins confortablement les frontières de cette dernière.

L'approche supranationale reconnaît donc que les identités nationales dominent le paysage identitaire européen et que cette situation ne risque pas de se modifier de façon spectaculaire dans un avenir rapproché. Mais on croit fermement qu'une identité européenne peut venir s'ajouter au spectre identitaire déjà remarquablement diversifié des individus et des collectivités européennes. Cette notion de somme ou de multiplicité des identités est un thème récurrent de la littérature supranationale.

Les auteurs proposent deux voies principales qui créeraient un contexte favorable à l'émergence d'une identité européenne, à moyen ou à long terme. D'une part, on estime qu'on doit poursuivre les efforts de symbolisation mis en oeuvre par l'Europe et par les gouvernements des États membres depuis quelques années, par exemple la valorisation de l'Europe et de son histoire dans les systèmes d'éducation nationaux, l'apparition du drapeau, l'utilisation d'un passeport commun, et l'implantation de réseaux non-économiques comme les échanges étudiants et scientifiques. D'autre part, la citoyenneté européenne est également perçue comme un moyen effectif de générer un sentiment d'appartenance à une communauté «imaginée» de semblables (Grant 1998, p. 160).

Ceci nous amène à remarquer en dernier lieu que la façon supranationale de concevoir les conditions de la légitimité des institutions européennes, c'est-à-dire par la nécessité de l'existence d'une communauté d'Européens, est remarquablement semblable à la façon dont la mise en place de l'État-nation est justifiée et expliquée. II apparaît qu'ainsi, la définition de l'identité européenne qui est avancée se rapproche de celle qui est généralement attribuée à l'identité nationale?

2. Voir à cet égard, l'ouvrage de Dominique Schnapper qui nous apparaît particulièrement pertinent à l'étude du concept de nation, par son caractère clair et nuancé (Schnapper 1994, p. 27-38). 


\section{La trop grande fragilité du concept de culture européenne}

Une des premières critiques qui vient à l'esprit à l'analyse de la proposition supranationale est qu'il est difficile de voir, dans le cas de la préexistence d'un fonds culturel commun, où se situent précisément les pays d'Europe de l'Est qui ont postulé pour devenir membre de l'union, et dont les candidatures font actuellement l'objet d'un examen sérieux. Cet aspect de la définition de la conscience européenne pourrait en fait se révéler être singulièrement épineuse si la question du statut européen de la Turquie venait également à l'ordre du jour. D'autant plus que si l'on pousse la logique encore plus loin, les sociétés nord-américaines feraient plus aisément partie d'une civilisation européenne que la Russie, par exemple.

Malgré le fait que ce type de définition de l'identité semble correspondre adéquatement à la réalité, postuler un passé historique et civilisationnel commun à l'ensemble de ce continent est donc risqué et ne résisterait sans doute pas à un examen sérieux. Les sociétés européennes ont une lecture de ce passé commun qui est d'abord et avant tout nationale et qui, dans cette perspective, est susceptible de mener à des compréhensions divergentes du même phénomène.

The incongruencies in the images of the same historical event between the European nations can, for instance, be seen in the contradictory interpretation that the same historical personality receives in the different european nations. The English worship Sir Francis Drake as a hero of liberty, whereas the Spaniards see him as a pirate and international criminal who broke the law of the European nations (Koslowski 1998, p. 223).

Ceci nous amène à un deuxième commentaire majeur. Même si l'approche supranationale attribue à l'identité nationale une place importante dans le cadre de la future Europe, elle n'est pas très explicite quant à la façon dont cette dernière pourrait éventuellement s'agencer avec la conception de l'identité européenne qui est préconisée. En fait, le lecteur a un peu l'impression de poupées russes lorsqu'il se retrouve devant ce type de proposition, c'est-à-dire devant l'idée plus ou moins implicite que les identités sont emboîtables les unes dans les autres et qu'une identité plus large viendra s'ajouter à toutes les autres, sans que cela ne pose de trop grandes difficultés.

Il est intéressant de noter que cette représentation de la réalité est souvent décrite par les concepts d'identités "à multiples facettes" 
(multifaceted) ou encore, "multi-étagées" (multilayered), qui sont amenés comme tenant de l'évidence et s'en trouvent ainsi très peu définis ce qui, à notre avis, présente une valeur explicative limitée. Affirmer la complexité d'un phénomène n'aide pas à sa compréhension, elle ne lui attribue qu'une caractéristique. Lorsque, par exemple, Meehan affirme qu'une nouvelle sorte de citoyenneté est en train d'émerger en Europe et que cette dernière s'exprime à travers une configuration de plus en plus complexe de réseaux, d'alliances volontaires et d'institutions communes, selon nous, elle n'explique pas ce qu'est l'identité européenne ou la citoyenneté, mais procède à une description plutôt vague des conditions d'émergence et d'expression identitaire, qui pourraient même s'appliquer à d'autres lieux que l'Europe (Meehan 1993, p. 1).

En dernier lieu, nous aimerions souligner le fait que la manière dont les auteurs abordent la question de l'identité européenne, c'està-dire comme nécessaire à la légitimation d'institutions qui existent et agissent déjà dans la réalité, nous paraît établir des liens un peu mécaniques et fonctionnels entre institutions et identité. Comme nous l'avons souligné au début de ce travail, les lacunes démocratiques de l'Europe font l'objet d'une critique qui est plutôt récente alors que la plupart de ces institutions existent depuis plusieurs dizaines d'années. Par ailleurs, si l'on se risque à faire un peu de prospective, on peut se demander, dans le cas de l'irruption d'une crise ou d'un litige majeur entre les pays membres, qu'elles seraient les institutions ou encore les identités qui seraient les plus susceptibles de rallier les diverses populations, surtout celles en opposition.

\section{L'Europe des régions - l'Europe des nations: le nouveau visage du continent}

Nous aimerions préciser d'entrée de jeu que l'approche que nous avons intitulée "l'Europe des régions" est la plus éclatée de toutes celles abordées jusqu'à présent dans cet article. En effet, même s'il est possible de regrouper les auteurs logeant sous cette enseigne en fonction du fait qu'ils présentent tous une image extrêmement complexe de l'Europe, et du fait qu'ils mettent l'accent sur un phénomène de fragmentation de l'espace européen et de sa territorialité, il demeure néanmoins difficile de dégager de leurs discours une approche cohérente et structurée en tant que telle.

Nous notons toutefois que ce que nous avons regroupé comme étant l'Europe des régions montre deux dimensions distinctes. Une première catégorie d'analystes s'intéresse plus particulièrement à la 
résurgence des identités régionales et sera portée à examiner minutieusement le cas d'une région, par exemple. La plupart du temps, les régions choisies sont celles dont les revendications autonomistes sont bien connues : la Corse, l'Écosse, le pays Basque ou la Catalogne. Un second groupe s'attache plus particulièrement à tenter de décrire et de prévoir ce que sera le paysage politico-économique européen face à une multiplication des acteurs occasionnée par le phénomène de régionalisation et d'érosion des frontières nationales à l'intérieur de l'Europe. Ces deux façons différentes d'aborder la question font en sorte qu'il est difficile de dégager une vue d'ensemble. II est toutefois possible de retenir quelques point de repères d'une lecture qui rallie un nombre important d'auteurs.

De façon générale, on part du constat que le processus de construction européen, au lieu d'inciter à un transfert des allégeances vers un regroupement d'intérêts ou de sentiments encore plus large que celui provoqué par l'appartenance nationale, aurait concrètement entraîné le phénomène inverse et provoqué un mouvement vers les petits ensembles ainsi qu'un fractionnement et une complexification de l'espace de participation politique. On part donc d'un certain constat que le territoire et la souveraineté ne correspondent plus et l'on tente d'expliquer cette dynamique en regard des nouveaux paramètres établis par l'existence de l'Europe.

La plupart des auteurs soulignent dès le départ que ce sont les institutions européennes qui ont conceptualisé en termes régionaux la diversité caractérisant leurs membres (Graham et Hart 1999, p. [3]). Les programmes régionaux d'harmonisation et convergence, ciblant avant tout des objectifs économiques, ont réintroduit la région comme acteur dans le paysage politique en lui conférant un rôle prépondérant au plan de l'orientation du développement et de la croissance économique. Ce faisant, l'UE, au lieu d'avoir déterritorialisé la pratique politique (Smouts 1998, p. 37), aurait au contraire marqué la relation de ses membres avec leur territoire, qui est ici plus parcellaire que celui de l'État-nation.

The new Europe of the EC is reconstructing its societies into a myriad of smaller but no less complex "new Europes", sociocultural groupings and configurations which face the tensions between tradition and change as well as the disconcerting choice of whether, or how to influence, curtail or stop the processes of EC political and economic integration (Wilson 1993, p. 2).

Vus sous cet angle, les programmes régionaux de l'Union 
européenne auraient permis à plusieurs «identités régionales marginalisées", de se réaffirmer et de reformuler leurs intérêts en fonction d'un nouvel espace où elles peuvent désormais s'exprimer comme collectivité. Cette situation aurait donc permis une redéfinition du lien que ces communautés entretiennent avec le pouvoir central de l'État dont elles font partie. Ces identités régionales, sont, par ailleurs, perçues par la plupart des analystes comme étant plus anciennes que les identités nationales proprement dites (Smouts 1998, p. 30). L'Europe, en accordant à l'acteur régional une représentation politique plus significative et, surtout, un accès à des ressources financières substantielles, aurait donc entraîné des modifications importantes dans la relation qui existait auparavant entre les pouvoirs centraux des États membres et leur périphérie.

Plaçant essentiellement au centre de l'analyse les enjeux économiques, la plupart des régionalistes estiment que le phénomène de régionalisation auquel l'Europe a contribué illustrerait l'incapacité de l'État-nation occidental traditionnel à répondre à l'enjeu de la mondialisation.

The political implications of this geographical reframing of center-periphery relations are clear. European integration is a sacrifice of national autonomy in the context of global power relations which make Europe vulnerable and can be seen as a response to the growing inadequacies of the Western European Nation State (Jaffe 1993, p. 64).

La régionalisation est considérée ici comme un phénomène assumant principalement deux fonctions. En premier lieu, elle permet aux régions d'affirmer la prédominance d'une identité plus vieille et plus "adéquate» que celle qui aurait été imposée par l'État-nation; l'accès à des ressources autres que celles du pouvoir central, leur permet également de redéfinir les termes des relations souvent problématiques et inégales qu'elles entretenaient avec le pouvoir central (Jaffe 1993). En second lieu, la région, même si elle se situe à cheval sur une frontière nationale, constituerait une entité plus "naturelle» d'échanges commerciaux et économiques, assurant mieux la croissance et la prospérité des populations qui la composent que ne le fait l'État-nation.

As borders loose their meaning, deeply rooted patterns of commercial and cultural interaction are reappearing in regions where people have more in common, culturally or economically, with neighbors across the border than with their fellow countrymen (Newhouse 1997, p. [4]). 
L'unification politique est donc envisagée sous un angle complètement différent de ceux privilégiés par les approches postnationale et supranationale. Ici, la nécessité du lien entre identité européenne et intégration n'est pas posée. Au contraire, on avance plutôt que l'Europe doit demeurer une organisation fonctionnelle, rationnelle et stratégique capable de garantir la prospérité économique des régions et l'épanouissement des cultures régionales qui doivent saisir cette occasion inespérée offerte par l'affaiblissement des institutions nationales pour rétablir leurs schémas traditionnels d'échanges et souvent renouer avec la prospérité.

Ce que les tenants de l'approche régionaliste présentent est donc une image extrêmement fragmentée d'une identité européenne qui est perçue comme une architecture complexe et mouvante des acteurs et de leurs identités (régions, États, UE, corporations, groupes de pression, etc.), de leurs interactions, de leurs alliances et de leurs conflits au sein d'un territoire et d'un espace dont les frontières sont fluides et en constante redéfinition (Graham et Hart 1999, p. [11]). Dans cette perspective, on attribue à l'Europe le rôle d'un semi-État aux caractéristiques proprement post-modernes, dans la mesure où elle se distingue de plus en plus des formes modernes nationales de gouvernance.

(Anderson) observes that the EU has been characterized as perhaps the world's first truly post-modern international political entity, distinct from the national and federal state forms of the modern era, but in some respect reminiscent of pre-modern territorialities (Graham et Hart 1999, p. [11]).

Protecteur des minorités nationales à l'interne et "forteresse" contre les effets négatifs de la mondialisation à l'externe (Laitin 1997, p. [10]), cet État n'aurait besoin d'aucune identité pour asseoir sa légitimité. Celle-ci passerait en effet par une reconnaissance rationnelle de la part de tous les acteurs qu'elle est la seule instance, dans le contexte qui prévaut actuellement sur la planète, capable de représenter un intérêt commun défini en termes quasi exclusivement économiques (Imbert 1993, p. 40).

À travers la littérature, nous avons relevé que la définition de l'identité suggérée par les tenants de l'approche régionale privilégie ses dimensions culturelles et ethniques, aspects qui ont été vigoureusement rejetés par les deux approches examinées précédemment. Le simple fait d'affirmer le caractère plus "naturel" ou moins coercitif des identités régionales, qui sont présentées comme 
ayant une existence pré-moderne, donc préalable à celle des identités nationales, suggère que l'identité est essentiellement un fait de culture et de traits partagés par une collectivité, par exemple une langue, une religion, un système de valeurs, des coutumes et des rituels, etc. Cette dimension permet à la fois de lidentifier comme une collectivité, de générer un sentiment d'appartenance et de la distinguer d'autres collectivités dont les caractéristiques et les traits communs diffèrent (Parman 1993, p. 191).

En dernier lieu, il est intéressant de noter que les auteurs de l'approche régionaliste semblent peu préoccupés par la question des nationalismes d'extrême droite. La résolution de cette problématique est plutôt implicite dans leurs discours. Certains croient fermement que l'interdépendance économique qui existe déjà depuis plusieurs années, si elle n'élimine pas complètement les risques de conflits armés, aurait tendance à décourager la violence puisque la prospérité dépendrait du maintien des échanges et de l'ouverture des frontières. D'autres estiment que l'Europe, en offrant aux régions un accès aux ressources, une reprise du contrôle sur les orientations du développement économique ainsi qu'un forum pour faire entendre leurs revendications, rend inutile le recours à la violence pour s'affirmer (Parman 1993, p. 189).

\section{Une vision instrumentale et un rôle limité pour l'identité}

Dans l'optique privilégiée par les tenants de l'approche régionaliste, l'Europe semble perçue en termes quasi exclusivement instrumentaux et elle ne serait en fait qu'une composante d'un processus de redéfinition vaste et complexe de l'espace d'échange économique, de revendication et de représentation politique, auquel elle contribue par ses décisions et ses actions, mais qu'elle ne commande pas. Le questionnement quant à l'identité européenne est présentée, plus souvent qu'autrement, comme étant une préoccupation secondaire face aux phénomènes d'ordre économique qui s'imposent au continent et qui sont eux, les principaux responsables de sa fragmentation et, par effet d'entraînement, des identités. C'est pourquoi il est difficile de discerner la définition de l'identité européenne proposée par les auteurs régionalistes, ceux-ci préférant situer ce questionnement au niveau des plus petits ensembles plutôt que des grands. Mais lus sous cet angle, les auteurs de l'école régionaliste réussissent à rendre compte d'un phénomène que les autres approches évacuent complètement de l'analyse, soit celui de la résurgence et de la réaffirmation d'identités "sous-nationales". 
Nous trouvons toutefois que le caractère instrumental et fonctionnel qui est attribué à une Europe trouvant sa raison d'être soit dans la protection des minorités, soit dans la garantie d'un avenir économique prospère, est un peu réducteur de ce qu'est l'Europe, de son rôle et de son influence sur les sociétés européennes et de ce qu'elle pourrait représenter pour ces dernières. À l'instar des postnationaux, une identité fondée uniquement sur des critères rationnels qui dans le cas présent, reposent surtout sur une dimension économique, n'apparaît pas satisfaisante et ne semble pas établir adéquatement en quoi doit consister une identité européenne significative ni lui attribuer un rôle qui dépasserait l'instrumentation. Par ailleurs, la prédominance accordée à la dimension économique nous paraît également biaiser l'approche car les contingences qu'elle impose y ont un caractère inévitable, voire des allures de processus naturel alors qu'en fait, elles représentent le fruit de décisions humaines. C'est pourtant cet aspect négligé de la réalité par les auteurs qui est au coeur des questions de démocratie, de représentativité et de légitimité qui sont toute à fait cruciales actuellement dans le débat sur l'identité européenne.

Une autre remarque importante qui vient à l'esprit lorsqu'on tente de synthétiser l'approche régionale concerne le fait que les auteurs semblent vouloir appliquer à l'échelle régionale le principe territorial de souveraineté qui a longtemps justifié l'emprise de l'État-nation sur les régions. La définition qui est donnée à l'identité des régions lui confère des dimensions plus ethniques ou culturelles, telles la langue, les coutumes, le sentiment d'une histoire et d'un destin partagé: ces éléments justifient la défense des intérêts régionaux, un peu comme l'existence de la nation avait permis la formulation et souvent, la domination de l'intérêt national sur toute autre considération. Ne s'agirait-il pas, dans le cas de l'Europe, d'une façon un peu étroite de concevoir l'identité?

\section{L'impossible identité européenne : la critique fondamentale d'Anthony D. Smith}

Dans un dernier temps, il semble important de rapporter ici un point de vue qui, sans constituer une véritable approche, n'en demeure pas moins la dernière position significative dans le débat actuel sur l'identité européenne. Dans un article au contenu remarqué publié dans la revue International Affairs en 1992, Anthony D. Smith fait le tour du débat d'une façon très critique pour en arriver à la conclusion que l'émergence d'une identité européenne telle qu'elle a été définie jusqu'à présent n'est ni possible, ni souhaitable. Ses 
principaux arguments ont, depuis la parution de ce texte, été repris et approfondis par d'autres auteurs, par ailleurs moins nombreux que dans les autres approches, qui rejettent eux aussi de façon critique les diverses conceptions de l'identité européenne qui ont été avancées jusqu'à présent. Toutefois, aucun n'est aussi explicite que Smith lui-même, et c'est pourquoi la majeure partie des références utilisées dans cette partie sont de lui.

L'objectif des interventions des "critiques" dans la discussion n'apparaît pas être de refuser totalement l'idée d'une identité européenne, mais plutôt de nuancer l'optimisme de ceux qui voient dans l'Europe les ingrédients permettant de reformuler sur de nouvelles bases à la fois l'identité politique, la participation, la légitimité et la démocratie. Smith et ses condisciples articulent donc leurs critiques en fonction de ce qui a été proposé dans la littérature et ils relèvent deux problèmes fondamentaux, soit celui de la conceptualisation de la diversité culturelle et celui de l'absence de mythes fondateurs et prémodernes à l'Europe.

Dans un premier temps, les critiques questionnent la façon dont la dimension culturelle - aspect qui est souvent situé au coeur des approches présentées dans ce travail - est abordée. D'une part, l'idée qu'il existerait un "fonds culturel commun" à l'ensemble des collectivités européennes tel qu'avancé par l'approche supranationale, semble créer un malaise particulièrement aigu, surtout chez Smith. Plus précisément, il questionne sérieusement ce présupposé de la définition de l'identité européenne, qu'il qualifie de "formule officielle", le considérant inapproprié à la définition de l'identité. Sans remettre en cause les diverses traditions partagées en Europe, tel l'humanisme et l'État de droit par exemple, il estime que de là à postuler une culture commune, il y a un pas trop important à franchir.

What are these partially shared traditions and heritages? (...) Together they constitute not a "unity in diversity" - the official European cultural formula - but a family of cultures made up of a syndrome of partially shared historical traditions and cultural heritages (Smith 1992, p. 70).

Cette "famille de culture" ne serait donc pas suffisante pour faire émerger un sentiment d'appartenance à l'Europe, au même titre que la nation réussit à le faire, par exemple. Dans cette perspective, les critiques réfutent l'idée qu'il puisse y avoir un sentiment d'appartenance européen et soulignent, pour appuyer leur point de vue, que le processus de construction européen est avant tout affaire de 
rationalité puisqu'il a surtout été pensé en fonction de critères concernant la sécurité et la prospérité du continent.

De plus, la plupart des auteurs notent que malgré les efforts de socialisation et de mise en valeur de ce qui est commun et que malgré les diverses politiques adoptées, la fragmentation sociale et l'hétérogénéité culturelle sont encore ce qui caractérise le plus adéquatement le continent (Obradovic 1996, p. 213-214). Gireault (1994) constate lui aussi l'absence d'une identité européenne et souligne le fait que l'identification la plus importante, à l'échelle du continent, $y$ est encore nationale.

Par ailleurs, les auteurs qui adoptent cette approche critique répondent également à la conception largement répandue que les identités culturelles peuvent être multiples et qu'elles peuvent s'additionner, en relevant les principales difficultés et les questions que cette façon de concevoir l'identité soulèvent.

But if the possibility of being intensely French or British and intensely European exists, what does it mean to feel and be European? Is Europe merely the sum of its various national identities and communities? (...) On the other hand, if Europe and European signify something more than the sum total of the populations and cultures that happen to inhabit a conventionally demarcated geographical space, what exactly are those characteristics and qualities that distinguish Europe from anything or anyone else? (Smith 1992, p. 68).

Obradovic renchérit en signalant le fait que jamais la distinction entre identité nationale et identité européenne n'est effectuée, alors que distinguer l'une de l'autre, c'est-à-dire identifier ce qui relève de l'appartenance nationale et ce qui se rattache à un certain sentiment d'européanité permettrait sans doute de donner un contenu et une signification à la notion d'identité européenne (Obradovic 1996, p. 214). Pour l'ensemble des auteurs, l'inadaptation de ces conceptions de l'identité et du multiculturalisme est clairement illustrée par le fait que les divers États européens n'ont pas été capables de s'entendre sur une politique extérieure commune, exposant ainsi les divergences d'opinion publique des diverses nations et la primauté de l'intérêt national dans la conduite des affaires politiques.

Ces remarques, qui constituent le premier volet de l'approche critique, éclairent d'une façon assez directe les principales faiblesses des conceptions de l'identité européenne et, pour certaines, rejoignent nos propres observations. Mais les doutes quant à l'éventualité de 
l'émergence d'une identité européenne à proprement parler s'appuient également sur une autre dimension qui n'a été aucunement traitée auparavant, soit celle de l'absence de mythes fondateurs à l'Europe.

Cet argument est la pierre d'assise de la critique des auteurs. Rappelons que pour Smith, les mythes se retrouvent dans le passé pré-moderne des nations, passé qui fait particulièrement défaut à l'Europe actuelle. De plus, il est fort probable, selon lui, que plusieurs mythologies pré-modernes existent simultanément en Europe, alors que les modèles proposés d'une identité européenne privilégient systématiquement un ensemble de mythes qui correspond à des valeurs très spécifiques et surtout très actuelles (ouverture, tolérance, libre-arbitre, individualisme, etc.).

Dans cette perspective, comment sélectionner les mythologies qui seront au fondement de l'Européanité et celles qui n'y participeront pas? Smith suggère en fait qu'inévitablement, les mythes fondateurs de la majorité, c'est-à-dire ceux qui situent la primauté du mode de penser occidental, auront tendance à s'imposer et que cette seule éventualité constitue un frein considérable à l'établissement d'une identité collectivement et véritablement européenne et multiculturelle.

It is clear that such historical mythomoteurs are inappropriate for the modern European project. But where else can one look for the necessary political mythology? Is it possible for Europe to arise without myth and memory? Have we not seen that these are indispensable element in the construction of any durable and resonant collective cultural identity? Here lies the new Europe's true dilemma: a choice between unacceptable historical myths and memories on the one hand, and on the other a patchwork, memoryless scientific culture held together solely by the political will and economic interest that are so often subject to change (Smith 1992, p. 74).

Telles sont, de façon succincte, les principales critiques formulées par les tenants de "l'impossible" (ou très peu probable?) identité européenne. Nous aimerions également souligner le fait que Raymond Aron avait également émis des doutes quant au sens attribué à l'espace politique que représente l'Europe pour ses membres, et ce, en 1974. Indirectement, sa principale préoccupation concernait l'identité et se penchait sur la question de la possibilité de la coexistence de plusieurs citoyennetés en sol européen. En effet, pour lui, le fait qu'il existe simultanément plusieurs citoyennetés en 
Europe «affaiblit plutôt qu'il ne renforce l'expérience vécue de la citoyenneté politique. Le citoyen sait de moins en moins qui prend les décisions, si elle sont prises à Bruxelles ou dans la capitale de la nation" (Aron 1991-1992 [1974], p. 201). Cette remarque paraît encore pertinente à l'heure actuelle, où l'un des principaux reproches fait à l'Europe est son manque de transparence et sa complexité bureaucratique.

\section{Le piège de la définition de l'identité selon Smith}

Smith est un des rares auteurs à avoir tenté d'intégrer la dimension affective au sein d'une définition de l'identité et à avoir voulu expliquer l'origine du sentiment d'appartenance qui semble être une dimension primordiale de l'identité. Toutefois, cette dimension constitue également la principale faiblesse de toute son argumentation. Le fait est que la critique repose essentiellement sur une définition que Smith avait élaborée pour expliquer l'identité nationale. Cette définition avance que :

Identities are forged out of shared experiences, memories and myths, in relation to those of other collective identities. They are in fact forged through opposition to the identities of significant others, as the history of paired conflicts so often demonstrates (Smith 1992, p. 75).

L'identité, pour Smith, repose donc à la fois sur le sentiment d'un passé et d'une mythologie partagée ainsi que sur certains éléments qui permettent une différentiation par rapport à d'autres collectivités. Ces éléments sont, quant à eux, étroitement associés à des dimensions culturelles telles la langue et les coutumes et trouvent également leur origine dans le passé pré-moderne de la collectivité, dans ce cas-ci, la nation (Rakic 1998, p. [3]).

Or, l'idée implicite qui soutient le débat sur l'identité européenne est précisément de tenter de formuler une définition de l'identité qui serait différente de celle de l'identité nationale, puisque la majeure partie des auteurs sont conscients, selon nous, que ce type d'identification ne serait sans doute pas appropriée à une entité politique telle que l'Europe. De plus, beaucoup d'analystes hésitent à attribuer à l'identité des caractéristiques culturelles puisqu'ils estiment que cette façon de la concevoir comporte beaucoup trop d'éléments problématiques et ne résiste pas, dans la plupart des cas, à une application empirique qui permet de découvrir qu'au sein d'une nation, il y a plusieurs identités, voire sous-nationalités. 
Nous aimerions également souligner en dernier lieu, que si l'on adopte, sans la modifier ou l'adapter, la définition de l'identité de Smith, on se doit de prendre une position critique, à l'égard de toute proposition de définition de l'identité qui s'articule sur des fondements différents de ceux qu'il reconnaît. Smith pose la nécessité d'une mythologie issue d'un passé pré-moderne, à la constitution d'une identité collective, ce qui dans le cas de l'Europe, apparaît immédiatement comme une difficulté immense. En effet, l'idée même d'une Europe unie économiquement et politiquement date véritablement, selon nous, de la deuxième moitié du XXe siècle et correspond à des conceptions particulièrement modernes d'impératifs de sécurité d'un territoire qui est encore déterminé par des frontières (que ce soit celles de l'Europe ou celles de l'État), ainsi que de rationalité économique par l'établissement d'un marché commun (Hensen et Williams 1999). Dans cette perspective, l'identité européenne est non seulement difficilement concevable, elle semble carrément impossible.

Conclusion : un débat qui révèle les difficultés conceptuelles de l'identité

Ce que l'élaboration de cette synthèse critique nous a permis de déceler en premier lieu est que l'élément central de la problématique de l'identité européenne, la dimension qui est systématiquement traitée par l'ensemble des approches examinées est celle de la difficulté posée par le pluralisme des sociétés européennes. La diversité culturelle semble être un élément particulièrement difficile à conceptualiser et à articuler avec la notion d'une seule identité européenne, tout en ne pouvant être niée compte tenu qu'il y a effectivement coexistence de plusieurs nations au sein du territoire couvert par l'Europe.

D'un bout à l'autre du spectre des idées proposées, ce qui semble susciter le plus de difficulté est donc précisément la conjonction entre la dimension affective de l'appartenance, l'identité et le multiculturalisme inhérent à l'Europe. Si l'on tente d'évacuer la dimension affective de l'identité, on aboutit à une conception qui apparaît plus qu'insatisfaisante de l'identité européenne; si l'on tente de la réintégrer totalement, on ne peut faire autrement qu'amener de sérieux doutes quant à l'émergence d'une identité européenne qui soit vraiment commune et également partagée. Dans tous les cas mitoyens, les définitions suggérées comportent des éléments d'exclusion qui apparaissent inacceptables dans le cadre européen. 
Peu importe quelle approche est privilégiée, les définitions de l'identité qui lui sont associées apparaissent donc systématiquement insatisfaisantes. Ce constat tend à laisser penser qu'il y a encore beaucoup de travail à effectuer sur cet aspect central et encore bien trop mal compris de l'organisation sociale et politique des collectivités et, selon nous, c'est précisément dans le creuset de débats comme celui portant sur l'identité européenne que la communauté scientifique sera capable de dégager une meilleure compréhension de ce phénomène.

Peut-être que les populations européennes ne seront jamais en mesure de dépasser la solidarité - calculée et rationnelle selon plusieurs - qui unit actuellement leurs nations. Ou, au contraire, peutêtre ne serons-nous capables d'identifier une identité européenne qu'une fois celle-ci déjà apparue et façonnée par les populations elles-mêmes, ce qui a tendance à être un peu oublié par les auteurs en termes de possibilités. On néglige souvent de considérer le rôle actif des populations dans la conduite de leur destin. Toujours est-il que le débat qui s'est installé autour de la notion d'identité européenne pose des questions fondamentales quant à la démocratie et quant à la représentativité d'un ensemble d'institutions qui agit directement dans les sociétés et qui influe sur la vie des gens. Dans cette optique, les réponses apportées et les solutions proposées doivent être suivies et leurs prémisses questionnées.

L'Europe comme nouvel espace politique et économique, l'Europe comme nouvelle pratique citoyenne, l'Europe comme nouvelle formulation des identités et des solidarités, peu importe l'angle sous lequel sont observés et interprétés les processus actuellement en cours sur le "vieux continent", le foisonnement d'idées que ceux-ci génèrent dans la communauté scientifique tend à laisser croire que les transformations actuelles affectant les vieux États nations pourront - peut-être? - aider à résoudre les pièges auxquels se heurtent les définitions de l'identité.

Aude-Emmanuelle Fleurant

Candidate au doctorat en science politique

Université du Québec à Montréal 


\section{Bibliographie et références}

ABÉLES, M., En attente d'Europe, Paris, Hachette, 1996.

ANDERSON, P., "Sous le signe du provisoire", dans Le Débat, no 91 (septembre-octobre 1996), p. 115-133.

ARON, R., "Une citoyenneté multinationale est-elle possible?", dans Commentaires (trad. d'un article paru en 1974 dans la revue Social Research), vol. 14, no 56 (1991-1992), p. 695-705.

BOOTH, W. J., "Communities of Memory : On Identity, Memory, and Debt", dans American Political Science Review, vol. 93, n० 2 (juin 1999), p. 249-263.

BULL, M. J., "Widening Versus Deepening the European Community :The Political Dynamics of 1992 in Historical Perspective", dans WILSON, T. M. et SMITH, E. M. (éds.), Cultural Change and the New Europe, Boulder (Col.), Westview Press, 1993, p. 25-45.

BUZAN, B., "Introduction : The changing security agenda in Europe», dans WAEVER, O. et al., Identity, Migration and the New Security Agenda in Europe, New-York, St-Martin's Press, 1993, p. 1-14.

BUZAN, B., "Societal Security, state security and internationalisation", dans ibidem, p. 41-58.

DELANTY, G., Inventing Europe. Idea, Identity, Reality, New-York, St-Martin's Press, 1995.

FEATHERSTONE, K., "Jean Monnet and the Democratic Deficit in the European Union vol. 32, no 2 (juin 1994), p. 149-170.

FERRY, J.-M., "Souveraineté et représentation", dans TÉLO, M. (éd.), Démocratie et construction européenne, Bruxelles, Éd. de l'Université de Bruxelles, 1995, p. 91-98.

GARCIA, S., "Europe's Fragmented Identities and the Frontiers of Citizenship", dans GARCIA, S. (éd.), European identity and the search for legitimacy, Londres, Pinter Publishers, 1993, p. 1-29.

GIREAULT, R. (dir.), Identité et conscience européennes au XXe siècle, Paris, Hachette, 1994.

GRAHAM, B. et HART, M., "Cohesion and Diversity in the European Union : Irreconcilable Forces?", dans Regional Studies, vol. 33, no 3 (mai 1999), p. 259-268. 
GRAHAM, B., "Introduction. Modern Europe : fractures and faults", dans GRAHAM, B. (éd.), Modern Europe. Place, Culture and Identity, Londres, Arnold Publishers, 1998, p. 1-15.

GRAHAM, B., "The past in Europe's present : diversity, identity and the construction of place", dans ibidem, p. 19-49.

GRANT, R., "The political geography of European Integration", dans ibidem, p. 145-163.

HANSEN, L. et WILLIAMS, M. C., "The Myths of Europe : Legitimacy, Community and the Crisis of the EU", dans Journal of Common Market Studies, vol. 37, no 2 (juin 1999), p. 233-249.

HABERMAS, J., "Citizenship and National Identity : Some Reflections on theFuture of Europe", dans BEINER, R. (éd.), Theorizing Citizenship, New-York, Presses de l'Université d'État de NewYork, 1995, p. 255-282

HART, M., "Convergence, cohesion and regionalism : contradictory trends in the new Europe", dans GRAHAM, B. (éd.), Modern Europe. Place, Culture and Identity, op. cit., p. 164-185.

HOWE, P., "A Community of Europeans : The Requisite Underpinnings", dans Joumal of Common Market Studies, vol. 33, n0 1 (mars 1995), p. 27-46.

IMBERT, C., "Identité européenne : le complexe de Prométhée", dans Fondation Robert Schuman (éd.), Les nouvelles frontières de l'Europe, Paris, Économica, 1993, p. 33-41.

JAFFE, A., "Corsican Identity and a Europe of Peoples and Regions", dans WILSON, T. M. et SMITH, E. M. (éds.), Cultural Change and the New Europe, op. cit., p. 61-80.

KEANE, J., "Nations, Nationalism and European Citizens", dans PERIWAL, S. (éd.), Notions of Nationalism, Budapest, Central European University Press, 1995, p. 182-207.

KOSLOWSKI, P., "Fatherland Europe? On European and National Identity and Democratic Sovereignty", dans FOLLESDAL, A. et KOSLOWSKI, P. (éds.), Democracy and the European Union, Berlin, Springer-Verlag, 1998, p. 214-230.

KYMLICKA, W. et NORMAN, W., "Return of the Citizen : A survey of Recent Work on Citizenship Theory", dans BEINER, R. (éd.), Theorizing Citizenship, op. cit., p. 283-322. 
LAFFAN, B., "The Politics of Identity and Political Order in Europe", dans Journal of Common Market Studies, vol. 34, no 1 (mars 1996), p. 81-102.

LAITIN, D. D., "The Cultural Identities of a European State", dans Politics and Society, vol. 25, no 3 (septembre 1997).

LEMKE, C., "Citizenship and European Integration", dans World Affairs, vol. 160, no 4 (printemps 1998), p. 212-217.

McCARTHY, P., "The Grand Bargain», Foreign Affairs, vol. 78, no 5 (septembre-octobre 1999), p. 150-157.

NEWHOUSE, J., “Europe's Rising Regionalism», dans Foreign Affairs, vol. 76, no 1 (janvier-février 1997), p. 67-84.

OBRADOVIC, D., "Policy Legitimacy and the European Union", dans Journal of Common Market Studies, vol. 34, no 2 (juin 1996), p. 191-221.

PARMAN, S., "The Future of European Boundaries : A Case Study", dans WILSON, T. M. et SMITH, E. M. (éds.), Cultural Change and the New Europe, op. cit., p. 189-202.

RAKIC, V., "Theories of Nation Formation and Case Selection: the Meaning of an Alternative Model', dans Nationalities Papers, vol. 26, no 4 (décembre 1998), p. 599-613.

REIF, K., "Cultural Convergence and Cultural Diversity as Factors in European Identity", dans GARCIA, S. (éd.), European Identity and the search for legitimacy. op. cit., p. 131-153.

SCHNAPPER, D., La communauté des citoyens. Sur l'idée moderne de nation, Paris, Gallimard, 1994.

SMITH, A. D., "National Identity ans the idea of European Unity", dans International Affairs, vol. 68, no 1 (1992), p. 55-76.

SMOUTS, M.-C., "The region as the new imagined community?", dans LE GALÈS, P. et LEQUESNE, C. (éds.), Regions in Europe, Londres, Routledge, 1998, p. 30-38.

TEMPLEMAN, S., "Constructions of Cultural Identity : Multiculturalism and Exclusion", dans Political Studies, vol. 47 (mars 1999), p. 17-31.

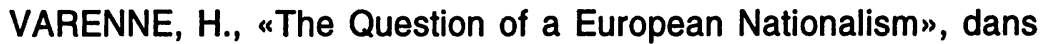
WILSON, T. M. et SMITH, E. M. (éds.), Cultural Change and the New Europe, op. cit., p. 223-240. 
WAEVER, O. et KELSTRUP, M., "Europe and its nations : political and cultural identities", dans WAEVER, $O$. et. al., Identity, Migration and the New Security Agenda in Europe, op. cit., p. 61-92.

WAEVER, O., "Societal Security : the concept", dans ibidem, p. 17-40.

WALLACE, W., "The Sharing of Sovereignty : the European Paradox", dans Political Studies, vol. 47, no 3 (numéro spécial, 1999), p. 503-521.

WIENER, A. et DELLA SALA, V., "Constitution-making and Citizenship Practice - Bridging the Democracy Gap in the EU?", dans Journal of Common Market Studies, vol. 35, no 4 (décembre 1997), p. 595-614.

WIENER, A., "Making sense of the new geography of citizenship : Fragmented citizenship in the European Union", dans Theory and Society, vol. 26 (1997), p. 529-560.

WILLIAMS, C. H., "Room to talk in a house of faith: on language and religion", dans GRAHAM, B. (éd.), Modern Europe. Place, Culture and Identity, Londres, Arnold Publishers, 1998, p. 186209.

WILSON, T. M., "An Anthropology of the European Community", dans WILSON, T. M. et SMITH, E. M. (éds.), Cultural Change and the New Europe, op. cit., p. 1-23.

WOLTON, D., La dernière utopie. Naissance de l'Europe démocratique, Paris, Flammarion, 1993, p. 69-132 et 405-451. 